\title{
Molecular biocoding of insulin
}

This article was published in the following Dove Press journal:

Advances and Applications in Bioinformatics and Chemistry

23 July 2010

Number of times this article has been viewed

\section{Lutvo Kurić}

Novi Travnik, Kalinska, Bosnia and Herzegovina

Correspondence: Lutvo Kurić 72290 Novi Travnik, Kalinska 7, Bosnia and Herzegovina

Tel +3876 I76 3917

Email lutvokuric@yahoo.com
Abstract: This paper discusses cyberinformation studies of the amino acid composition of insulin, in particular the identification of scientific terminology that could describe this phenomenon, ie, the study of genetic information, as well as the relationship between the genetic language of proteins and theoretical aspects of this system and cybernetics. The results of this research show that there is a matrix code for insulin. It also shows that the coding system within the amino acid language gives detailed information, not only on the amino acid "record", but also on its structure, configuration, and various shapes. The issue of the existence of an insulin code and coding of the individual structural elements of this protein are discussed. Answers to the following questions are sought. Does the matrix mechanism for biosynthesis of this protein function within the law of the general theory of information systems, and what is the significance of this for understanding the genetic language of insulin? What is the essence of existence and functioning of this language? Is the genetic information characterized only by biochemical principles or it is also characterized by cyberinformation principles? The potential effects of physical and chemical, as well as cybernetic and information principles, on the biochemical basis of insulin are also investigated. This paper discusses new methods for developing genetic technologies, in particular more advanced digital technology based on programming, cybernetics, and informational laws and systems, and how this new technology could be useful in medicine, bioinformatics, genetics, biochemistry, and other natural sciences.

Keywords: human insulin, insulin model, biocode, genetic code, amino acids

\section{Introduction}

The biologic role of any given protein in essential life processes, eg, insulin, depends on the positioning of its component amino acids, and is understood by the "positioning of letters forming words". Each of these words has its own biochemical base. If this base is expressed by corresponding discrete numbers, it can be seen that any given base has its own program, along with its own unique cybernetic and information characteristics. Indeed, the sequencing of the molecule is determined not only by distint biochemical features, but also by cybernetic and information principles. For this reason, research in this field deals more with the quantitative rather than qualitative characteristics of genetic information and its biochemical basis. For the purposes of this paper, specific physical and chemical factors have been selected in order to express the genetic information for insulin. Numeric values are then assigned to these factors, enabling them to be measured. In this way it is possible to determine if a connection really exists between the quantitative ratios in the process of transfer of genetic information and 
the qualitative appearance of the insulin molecule. To select these factors, preference is given to classical physical and chemical parameters, including the numbers of atoms in the relevant amino acids, their analog values, the position of these amino acids in the peptide chain, and their frequencies. There is a large number of these parameters, and each of them gives important genetic information. Going through this process, it becomes clear that there is a mathematical relationship between quantitative ratios and the qualitative appearance of the biochemical "genetic processes", and that there is a measurement method that can be used to describe the biochemistry of insulin.

\section{Methods}

Insulin can be represented by two different forms, ie, a discrete form and a sequential form. In the discrete form, a molecule of insulin is represented by a set of discrete codes or a multiple dimension vector. In the sequential form, an insulin molecule is represented by a series of amino acids according to the order of their position in the $1 \mathrm{AI} 0$ chains. Therefore, the sequential form can naturally reflect all the information about the sequence order and length of an insulin molecule. The key issue is whether we can develop a different discrete method of representing an insulin molecule that will allow accommodation of partial, if not all, sequence order information? Because a protein sequence is usually represented by a series of amino acid codes, what kind of numerical values should be assigned to these codes in order to optimally convert the sequence order information into a series of numbers for the discrete form representation? This section discusses how biochemical function of the molecule is determined by cybernetic information principles.

\section{Expression of insulin code matrix IAIO}

The matrix mechanism of insulin, evolution of biomacromolecules and, especially, biochemical evolution of the insulin language have been analyzed by the application of cybernetic methods, information theory, and system theory, respectively. The primary structure of a molecule of insulin is an expression of the exact specification of its atomic composition and the chemical bonds between those atoms.

\section{R6 insulin hexamer (dlai02)}

The structure $1 \mathrm{AI} 0$ has a total of 12 chains. Of these, two are sequence-unique identical chains of BFHJL (Figure 1).

\begin{tabular}{|l|l|}
\hline$>1 \mathrm{AI} 0:$ BFHJL & FVNQHLCGSHLVEALYLVCGERGFFYTPKT \\
\hline
\end{tabular}
Insulin B chain

Number of atoms

\begin{tabular}{|l|l|l|l|l|l|l|l|l|l|}
\hline F & V & N & Q & H & I & C & G & S & H \\
\hline 23 & 19 & 17 & 20 & 20 & 22 & 14 & 10 & 14 & 20 \\
\hline 1 & 2 & 3 & 4 & 5 & 6 & 7 & 8 & 9 & 10 \\
\hline
\end{tabular}

\begin{tabular}{|l|l|l|l|l|l|l|l|l|l|}
\hline L & V & E & A & L & Y & L & V & C & G \\
\hline 22 & 19 & 19 & 13 & 22 & 24 & 22 & 19 & 14 & 10 \\
\hline 11 & 12 & 13 & 14 & 15 & 16 & 17 & 18 & 19 & 20 \\
\hline
\end{tabular}

\begin{tabular}{|l|l|l|l|l|l|l|l|l|l|}
\hline $\mathrm{E}$ & $\mathrm{R}$ & $\mathrm{G}$ & $\mathrm{F}$ & $\mathrm{I}$ & $\mathrm{Y}$ & $\mathrm{T}$ & $\mathrm{P}$ & $\mathrm{K}$ & $\mathrm{T}$ \\
\hline 19 & 26 & 10 & 23 & 22 & 24 & 17 & 17 & 24 & 17 \\
\hline 21 & 22 & 23 & 24 & 25 & 26 & 27 & 28 & 29 & 30 \\
\hline
\end{tabular}

Rank $=1,2,3, \mathrm{n}$

Figure I Number of atoms in insulin B chain.

Notes: And in that dimension we can find an explanation for the given empirical reality. Aforementioned amino acids are positioned from numbers I to 30. Numbers I,2,3,n... present the position of a certain amino acid.

This positioning is of key importance for understanding the programmatic, cybernetic, and information elements of this protein. The scientific key for interpretation of biochemical processes is the same for insulin as for other proteins and sequences in biochemistry. The first amino acid in this example has 23 atoms, the second 19, the third 17, etc. They have exactly these numbers of atoms because there are many codes in the insulin molecules, analog codes, and other coded features. In fact, there is a cybernetic algorithm in which it is "recorded" that the first amino acid has to have 23 atoms, the second 19, the third 17, etc. The first amino acid has its own biochemistry, as does the second and the third, etc. The obvious conclusion is that there is a concrete relationship between quantitative ratios in the process of transfer of genetic information and qualitative appearance, ie, the characteristics of the organism.

\section{Bio insulin code}

The bio insulin code is an area of biomacromolecular processes in biochemistry (chemical engineering, bioprocess engineering, information technology, biorobotics) that treats signals as stochastic processes, dealing with their biosignal properties (eg, frequencies, mean, and covariance). In this context, biocodes are modeled as functions consisting of both deterministic and stochastic components. A simple example and also a common model of many bio systems is a signal Bio ( $t$ ) that consists of a deterministic part $\mathrm{x}(\mathrm{t})$ as a white biocode (Figure 2).

Bio $(\mathrm{t})=[\mathrm{X} 1,2,3, \mathrm{n}(\mathrm{t})-\mathrm{X} 1,2,3, \mathrm{n}(\mathrm{t})]$

$X(t)=$ Biocode 1,2,3,n 
$(\mathrm{A}, \mathrm{B}, \mathrm{C})-(\mathrm{B}, \mathrm{C}, \mathrm{D})=$ biocode 1

$(\mathrm{B}, \mathrm{C}, \mathrm{D}-\mathrm{C}, \mathrm{D}, \mathrm{E})=$ biocode 2

$(\mathrm{D}, \mathrm{E}, \mathrm{F}-\mathrm{E}, \mathrm{F}, \mathrm{G})=$ biocode 3 (1) etc.

where $\mathrm{A}, \mathrm{B}, \mathrm{C}$, and $\mathrm{n}$ represent the connection of group amino acid positions, 1,2,3,n

\begin{tabular}{|l|l|l|l|l|l|}
\hline $\mathrm{F}$ & $\mathrm{V}$ & $\mathrm{N}$ & $\mathrm{Q}$ & $\mathrm{H}$ & $\mathrm{I}$ \\
\hline 23 & 19 & 17 & 20 & 20 & 22 \\
\hline 1 & 2 & 3 & 4 & 5 & 6 \\
\hline
\end{tabular}

$$
\begin{gathered}
\operatorname{Bio}(\mathrm{t})=(\mathrm{F}, \mathrm{V}, \mathrm{N})-(\mathrm{Q}, \mathrm{H}, \mathrm{I})>(23,19,17)-(20,20,22) \\
\downarrow \\
(231917-202022)=29895
\end{gathered}
$$

Figure 2 Fragment of insulin $B$ chain.

Notes: The connection of the groups of these amino acids gives us number 29895 as a result. That was the code that represent one of the quantitative characteristics of the given genetic information.

Thus, the union of these amino acids generates the number 29895 , which is a code representing one of the quantitative characteristics of the given genetic information. In a similar way we can calculate biocodes for other groups of amino acids, which are connected by various biocodes and analog codes, as well as other quantitative features. Connection is one of the numerical expressions that connect various corresponding features in biochemistry. It has a very prominent place in our mathematical understanding of all processes in biochemistry. This is a recently discovered phenomenon, the role and significance of which will hopefully be clarified in the future. Those bioprocesses are well correlated, and the autocorrelation function is a biocode:

Bio $\mathrm{I}=(\mathrm{X} 1,2,3, \mathrm{n}(\mathrm{t})-\mathrm{X} 1,2,3, \mathrm{n}(\mathrm{t}))=[(-) \mathrm{Y} \leftrightarrow(+) \mathrm{Y}]$ $[(-) \mathrm{Y}=(+) \mathrm{Y}](2)$

where $\mathrm{Y}$ represents the result as a functional biocode.

\section{Insulin B chain}

Mathematical evidence is provided here to prove that in the biochemistry of insulin there really is a programmatic and cybernetic algorithm in which it is "recorded", in the language of mathematics, how the molecule will be built and what will be the quantitative characteristics of the given genetic information.

$\operatorname{Bio}(\mathrm{t})=\mathrm{X} 1,2,3, \mathrm{n}(\mathrm{t})-\mathrm{X} 1,2,3, \mathrm{n}(\mathrm{t})$

Where:

$\mathrm{X}(\mathrm{t})=$ biocodes $1,2,3, \mathrm{n}$

Bio $1=(\mathrm{A}, \mathrm{B}, \mathrm{C})-(\mathrm{B}, \mathrm{C}, \mathrm{D})=$ biocodes 1

Examples are presented in Figure 3.

Biocode 1

\begin{tabular}{|c|c|c|c|c|c|c|}
\hline $\mathrm{F}$ & $\mathrm{V}$ & $\mathrm{N}$ & $\mathrm{Q}$ & $\mathrm{H}$ & $\mathrm{I}$ & $\mathrm{C}$ \\
\hline 23 & 19 & 17 & 20 & 20 & 22 & 14 \\
\hline 1 & 2 & 3 & 4 & 5 & 6 & 7 \\
\hline $\boldsymbol{У}$ & $\downarrow$ & $\boldsymbol{K}$ & $\downarrow$ & $\mathbf{y}$ & $\downarrow$ & $\mathbf{K}$ \\
\hline & 59 & & 20 & & 56 & \\
\hline
\end{tabular}

$\downarrow$

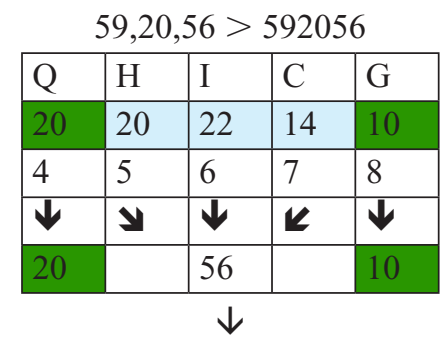

$20,56,10>205610$

Bio $1=(592056-205610)=386446$

Biocode 2

\begin{tabular}{|l|l|l|l|l|l|l|}
\hline $\mathrm{H}$ & $\mathrm{I}$ & $\mathrm{C}$ & $\mathrm{G}$ & $\mathrm{S}$ & $\mathrm{H}$ & $\mathrm{L}$ \\
\hline 20 & 22 & 14 & 10 & 14 & 20 & 22 \\
\hline 5 & 6 & 7 & 8 & 9 & 10 & 11 \\
\hline $\boldsymbol{У}$ & $\downarrow$ & $\boldsymbol{K}$ & $\downarrow$ & $\mathbf{У}$ & $\downarrow$ & $\boldsymbol{K}$ \\
\hline & 56 & & 10 & & 56 & \\
\hline
\end{tabular}

$\downarrow$

\begin{tabular}{|l|l|l|l|l|}
\multicolumn{5}{c|}{$56,10,56>561056$} \\
\hline $\mathrm{G}$ & $\mathrm{S}$ & $\mathrm{H}$ & $\mathrm{L}$ & $\mathrm{V}$ \\
\hline 10 & 14 & 20 & 22 & 19 \\
\hline 8 & 9 & 10 & 11 & 12 \\
\hline$\downarrow$ & $\mathbf{y}$ & $\downarrow$ & $\boldsymbol{K}$ & $\downarrow$ \\
\hline 10 & \multicolumn{5}{|c|}{} & 56 & & 19 \\
\hline \multicolumn{7}{|c|}{$\downarrow$}
\end{tabular}

$10,56,19>105619$

Bio $2=(561056-105619)=455437$

Biocode 3

\begin{tabular}{|l|l|l|l|l|l|l|}
\hline $\mathrm{S}$ & $\mathrm{H}$ & $\mathrm{L}$ & $\mathrm{V}$ & $\mathrm{E}$ & $\mathrm{A}$ & $\mathrm{L}$ \\
\hline 14 & 20 & 22 & 19 & 19 & 13 & 22 \\
\hline 9 & 10 & 11 & 12 & 13 & 14 & 15 \\
\hline $\mathbf{У}$ & $\downarrow$ & $\boldsymbol{K}$ & $\downarrow$ & $\mathbf{У}$ & $\downarrow$ & $\boldsymbol{K}$ \\
\hline & 56 & \multicolumn{7}{|c|}{} & 19 & & 54 & \\
\hline & $\downarrow$ & &
\end{tabular}

$56,19,54>561954$

\begin{tabular}{|l|l|l|l|l|l|l|}
\hline $\mathrm{V}$ & $\mathrm{E}$ & $\mathrm{A}$ & $\mathrm{L}$ & $\mathrm{Y}$ & $\mathrm{L}$ & $\mathrm{V}$ \\
\hline 19 & 19 & 13 & 22 & 24 & 22 & 19 \\
\hline 12 & 13 & 14 & 15 & 16 & 17 & 18 \\
\hline$\downarrow$ & $\mathbf{y}$ & $\Downarrow$ & $\boldsymbol{K}$ & $\mathbf{У}$ & $\downarrow$ & $\boldsymbol{K}$ \\
\hline 19 & & 54 & & & 65 & \\
\hline \multicolumn{7}{|c|}{$\downarrow$} \\
\hline
\end{tabular}

$19,54,65>195465$

Bio $3=(561954-195465)=366489$

Biocode 4

\begin{tabular}{|l|l|l|l|l|l|l|}
\hline E & A & L & Y & L & V & C \\
\hline 19 & 13 & 22 & 24 & 22 & 19 & 14 \\
\hline 13 & 14 & 15 & 16 & 17 & 18 & 19 \\
\hline $\mathbf{y}$ & $\downarrow$ & $\mathbf{K}$ & $\boldsymbol{У}$ & $\downarrow$ & $\mathbf{K}$ & $\downarrow$ \\
\hline & 54 & & & 65 & & 14 \\
\hline
\end{tabular}


$\downarrow$

$54,65,14>546514$

\begin{tabular}{|l|l|l|l|l|l|l|}
\hline $\mathrm{Y}$ & $\mathrm{L}$ & $\mathrm{V}$ & $\mathrm{C}$ & $\mathrm{G}$ & $\mathrm{E}$ & $\mathrm{R}$ \\
\hline 24 & 22 & 19 & 14 & 10 & 19 & 26 \\
\hline 16 & 17 & 18 & 19 & 20 & 21 & 22 \\
\hline $\boldsymbol{У}$ & $\downarrow$ & $\boldsymbol{K}$ & $\downarrow$ & $\mathbf{У}$ & $\downarrow$ & $\boldsymbol{K}$ \\
\hline & 65 & & 14 & & 55 & \\
\hline \multicolumn{7}{|c|}{} \\
& $\downarrow$ & &
\end{tabular}

$65,14,55>651455$

Bio $4=(546514-651455)=(-) 104941$

Biocode 5

\begin{tabular}{|l|l|l|l|l|}
\hline $\mathrm{C}$ & $\mathrm{G}$ & $\mathrm{E}$ & $\mathrm{R}$ & $\mathrm{G}$ \\
\hline 14 & 10 & 19 & 26 & 10 \\
\hline 19 & 20 & 21 & 22 & 23 \\
\hline$\downarrow$ & $\mathbf{y}$ & $\downarrow$ & $\boldsymbol{K}$ & $\downarrow$ \\
\hline 14 & & 55 & & 10 \\
\hline
\end{tabular}

$\downarrow$

$14,55,10>145510$

\begin{tabular}{|l|l|l|l|l|l|l|}
\hline $\mathrm{G}$ & $\mathrm{E}$ & $\mathrm{R}$ & $\mathrm{G}$ & $\mathrm{F}$ & $\mathrm{I}$ & $\mathrm{Y}$ \\
\hline 10 & 19 & 26 & 10 & 23 & 22 & 24 \\
\hline 20 & 21 & 22 & 23 & 24 & 25 & 26 \\
\hline $\boldsymbol{У}$ & $\downarrow$ & $\boldsymbol{K}$ & $\downarrow$ & $\boldsymbol{У}$ & $\downarrow$ & $\boldsymbol{K}$ \\
\hline & 55 & \multicolumn{6}{|c|}{} & 10 & & 69 & \\
\hline \multicolumn{7}{|c}{} \\
\hline
\end{tabular}

$55,10,69>551069$

Bio $5=(145510-551069)=(-) 405559$

Biocode 6

\begin{tabular}{|l|l|l|l|l|}
\hline $\mathrm{G}$ & $\mathrm{F}$ & $\mathrm{I}$ & $\mathrm{Y}$ & $\mathrm{T}$ \\
\hline 10 & 23 & 22 & 24 & 17 \\
\hline 23 & 24 & 25 & 26 & 27 \\
\hline$\downarrow$ & $\mathbf{y}$ & $\downarrow$ & $\boldsymbol{K}$ & $\downarrow$ \\
\hline 10 & \multicolumn{5}{|c|}{} & 69 & & 17 \\
\hline \multicolumn{5}{|c|}{$\downarrow$}
\end{tabular}

$10,69,17>106917$

\begin{tabular}{|l|l|l|l|l|l|l|}
\hline F & I & Y & T & P & K & T \\
\hline 23 & 22 & 24 & 17 & 17 & 24 & 17 \\
\hline 24 & 25 & 26 & 27 & 28 & 29 & 30 \\
\hline $\mathbf{У}$ & $\downarrow$ & $\boldsymbol{K}$ & $\Downarrow$ & $\mathbf{У}$ & $\Downarrow$ & $\boldsymbol{K}$ \\
\hline & 69 & & 17 & & 58 & \\
\hline
\end{tabular}

$\downarrow$

$69,17,58>691758$

Bio $6=(106917-691758)=(-) 681061$

Figure 3 Examples of biocodes.

Notes: The connection of the unions of these amino acids gives us number (-) 681061 as a result. That was the code that represent one of the quantitative characteristics of the given genetic information.

The biocodes presented in Figure 3 are calculated using the relationship between corresponding groups of amino acids. These are groups with different numbers of amino acids. There are different ways and methods of selecting these groups of amino acids, and it is hoped that science will soon determine which method is most efficient. Some biocodes have a positive numeric value and some have a negative one. Table 1 gives these codes (see also Figure 4).

Number of atoms

\begin{tabular}{|l|l|l|l|l|l|l|l|l|l|l|}
\hline $\mathrm{G}$ & $\mathrm{I}$ & $\mathrm{V}$ & $\mathrm{E}$ & $\mathrm{Q}$ & $\mathrm{C}$ & $\mathrm{C}$ & $\mathrm{T}$ & $\mathrm{S}$ & $\mathrm{I}$ & $\mathrm{C}$ \\
\hline 10 & 22 & 19 & 19 & 20 & 14 & 14 & 17 & 14 & 22 & 14 \\
\hline 1 & 2 & 3 & 4 & 5 & 6 & 7 & 8 & 9 & 10 & 11 \\
\hline
\end{tabular}

\begin{tabular}{|l|l|l|l|l|l|l|l|l|l|}
\hline S & L & Y & Q & L & E & N & Y & C & N \\
\hline 14 & 22 & 24 & 20 & 22 & 19 & 17 & 24 & 14 & 17 \\
\hline 12 & 13 & 14 & 15 & 16 & 17 & 18 & 19 & 20 & 21 \\
\hline
\end{tabular}

where amino acid $\mathrm{G}$ has 10 atoms, amino acids I 22 atoms, V 19 atoms, etc.

In this example there are 21 amino acids.

Rank $=1,2,3, \mathrm{n}$.

$\operatorname{Bio}(t)=[X 1,2,3, n(t)-X 1,2,3, n(t)](3)$

Where

$\mathrm{X}(\mathrm{t})=$ Biocodes $1,2,3, \mathrm{n}$

Figure 4 The formula for calculating of biocodes.

Notes: Aforementioned amino acids are positioned from number I to $2 \mathrm{I}$. The first amino acid in this example has 10 atoms, the second one 22, the third one 19 , etc.... Why do they have exactly this many atoms? There is a program-cybernetic algorithm in which it is "recorded" that the first amino acid has to have 10 atoms, the second one 22, the third one 19, etc... Mathematical expression for this algorithm are biocodes I,2,3,n.

Table I Overview of positive and negative values of biocodes for insulin chain B showing some of the quantitative characteristics of the insulin molecule and the exact mathematical balance between its components. Schematic representation of the biocode processing (I)

\begin{tabular}{|c|c|c|c|}
\hline $\begin{array}{l}\text { Biocodes } \\
I, 2,3, n\end{array}$ & $\begin{array}{l}\text { Biocodes } \\
I, 2,3, n\end{array}$ & $(I-2)(+)$ & $(I-2)(-)$ \\
\hline I & 2 & 3 & 4 \\
\hline 592056 & 205610 & Bio I = 386446 & - \\
\hline 205610 & 561056 & - & Bio $2=-355446$ \\
\hline 561056 & 105619 & Bio $3=455437$ & - \\
\hline 105619 & 561954 & - & Bio $4=-456335$ \\
\hline 561954 & 195465 & 366489 & - \\
\hline 195465 & 546514 & - & -351049 \\
\hline 546514 & 651455 & - & $-|0494|$ \\
\hline 651455 & 145510 & 505945 & - \\
\hline 145510 & 551069 & - & -405559 \\
\hline 551069 & 106917 & 444152 & - \\
\hline 106917 & 691758 & - & $-58484 \mid$ \\
\hline 691758 & 592056 & 99702 & - \\
\hline SUM & & $(+) 2258171$ & $(-) 225817 \mid$ \\
\hline
\end{tabular}


Biocode 1

\begin{tabular}{|l|l|l|l|l|l|}
\hline $\mathrm{G}$ & $\mathrm{I}$ & $\mathrm{V}$ & $\mathrm{E}$ & $\mathrm{Q}$ & $\mathrm{C}$ \\
\hline 10 & 22 & 19 & 19 & 20 & 14 \\
\hline 1 & 2 & 3 & 4 & 5 & 6 \\
\hline $\boldsymbol{y}$ & $\boldsymbol{\downarrow}$ & $\boldsymbol{K}$ & $\mathbf{Y}$ & $\boldsymbol{\Downarrow}$ & $\boldsymbol{K}$ \\
\hline \multicolumn{3}{|c|}{$10,22,19$} & \multicolumn{3}{c|}{$19,20,14$} \\
\hline
\end{tabular}

$\mathrm{GIV}=102219$

$\mathrm{EQC}=192014$

Bio $1=(\mathrm{GIV}-\mathrm{EQC})$

Bio $1=(102219-192014)=(-) 89795$

In this example we calculated the numbers of atoms in groups of three amino acids of the insulin chain.

Biocode 2

\begin{tabular}{|l|l|l|l|l|l|}
\hline E & Q & C & C & T & S \\
\hline 19 & 20 & 14 & 14 & 17 & 14 \\
\hline 4 & 5 & 6 & 7 & 8 & 9 \\
\hline $\boldsymbol{y}$ & $\boldsymbol{\downarrow}$ & $\boldsymbol{K}$ & $\mathbf{y}$ & $\downarrow$ & $\boldsymbol{K}$ \\
\hline \multicolumn{3}{|c|}{$19,20,14$} & \multicolumn{3}{|c|}{$14,17,14$} \\
\hline
\end{tabular}

$\mathrm{EQC}=192014$

$\mathrm{CTS}=141714$

Bio $2=(\mathrm{EQC}-\mathrm{CTS})$

Bio $2=(192014-141714)=(+) 50300$

Biocode 3

\begin{tabular}{|l|l|l|l|l|l|}
\hline C & T & S & I & C & S \\
\hline 14 & 17 & 14 & 22 & 14 & 14 \\
\hline 7 & 8 & 9 & 10 & 11 & 12 \\
\hline $\boldsymbol{y}$ & $\downarrow$ & $\boldsymbol{L}$ & $\mathbf{y}$ & $\downarrow$ & $\boldsymbol{K}$ \\
\hline \multicolumn{3}{|c|}{$14,17,14$} & \multicolumn{3}{c|}{$22,14,14$} \\
\hline
\end{tabular}

$\mathrm{CTS}=141714$

$\mathrm{ICS}=221414$

Bio $3=(\mathrm{CTS}-\mathrm{ICS})$

Bio $3=(141714-221414)=(-) 79700$ etc.

Biocodes are also shown in Table 2.

Figure 5 Examples of the connection of numbers of atoms in group of three amino acids of the insulin chain.

Notes: Biocodes I,2,3,n, represent one of the quantitative characteristics of the given genetic information.

\section{Analog biocode Insulin A chain}

Each numerical value has its analog expression. For example, the analog expression for number 19 is 91 (91 || 19). In a similar way we can calculate the analog expression for any numerical value (Figure 6). Our research has shown that analog codes are quantitative characteristics in biochemistry. An analog biocode is a discrete code that protects and guards genetic information coded in biochemical processes.
This is a recently discovered code, and more detailed knowledge about it is necessary.

Analog biocode 1

\begin{tabular}{|c|c|c|c|c|c|}
\hline $\mathrm{G}$ & I & $\mathrm{V}$ & $\mathrm{E}$ & Q & $\mathrm{C}$ \\
\hline 10 & 22 & 19 & 19 & 20 & 14 \\
\hline 1 & 2 & 3 & 4 & 5 & 6 \\
\hline$y$ & $\downarrow$ & $\boldsymbol{k}$ & $y$ & $\downarrow$ & $\boldsymbol{K}$ \\
\hline \multicolumn{3}{|c|}{$10,22,19$} & \multicolumn{3}{|c|}{$19,20,14$} \\
\hline \multicolumn{3}{|c|}{$\downarrow$} & \multicolumn{3}{|c|}{$\downarrow$} \\
\hline \multicolumn{3}{|c|}{ Analog code } & \multicolumn{3}{|c|}{ Analog code } \\
\hline \multicolumn{3}{|c|}{$\downarrow$} & \multicolumn{3}{|c|}{$\downarrow$} \\
\hline \multicolumn{3}{|c|}{912201} & \multicolumn{3}{|c|}{410291} \\
\hline
\end{tabular}

$$
\begin{aligned}
\mathrm{GIV} & =912201 \\
\mathrm{EQC} & =410291
\end{aligned}
$$

Analog biocode $1=(912201-410291)=(+) 501910$

Analog code of number 102219 is number 912201 (912201 || 102219)

Analog biocode 2

\begin{tabular}{|l|l|l|l|l|l|}
\hline $\mathrm{E}$ & $\mathrm{Q}$ & $\mathrm{C}$ & $\mathrm{C}$ & $\mathrm{T}$ & $\mathrm{S}$ \\
\hline 19 & 20 & 14 & 14 & 17 & 14 \\
\hline 4 & 5 & 6 & 7 & 8 & 9 \\
\hline $\mathbf{Y}$ & $\downarrow$ & $\boldsymbol{K}$ & $\mathbf{Y}$ & $\downarrow$ & $\boldsymbol{K}$ \\
\hline \multicolumn{2}{|c|}{$19,20,14$} & \multicolumn{3}{|c|}{$14,17,14$} \\
\hline \multicolumn{3}{|c|}{$\downarrow$} & \multicolumn{3}{|c|}{$\downarrow$} \\
\hline \multicolumn{3}{|c|}{ Analog code } & \multicolumn{3}{c|}{ Analog code } \\
\hline \multicolumn{3}{|c|}{$\downarrow$} & \multicolumn{3}{c|}{$\downarrow$} \\
\hline \multicolumn{2}{|c|}{41029141} \\
\hline
\end{tabular}

$\mathrm{EQC}=410291$

$\mathrm{CTS}=417141$

Analog bio $2=(410291-417141)=(-) 6850$, etc.

Figure 6 Examples of the analog codes of insulin chain A.

Notes: The analog expression for number 1920 I4 is 410291 , and number 14 I7 14 is 417 I4I. Analog code is, actually, a discrete code that protects and guards genetic information coded in biochemical processes.

Positive and negative analog codes of insulin chain A are shown in Table 3.

\section{Correlation between code and analog code}

Arithmetical expression for biocode $=190$ 001; there is a correlation between the code and analog code $=899809$; 100091|| $190001>89$ 910; 908998 || $899809>9189$. This phenomenon will be investigated into more detail in the future.

\section{Insulin model}

The structure $1 \mathrm{AI} 0$ has in total 12 chains, ie, A,B,C,D,E,F, $\mathrm{G}, \mathrm{H}, \mathrm{I}, \mathrm{J}, \mathrm{K}, \mathrm{L}$. In this group of chains there are three unions with four chains each. Each of these three groups of chains has an identical number of amino acids, identical number of atoms, and an identical sum of position numbers for these amino acids. 


\section{Groups A,B,K,L}

\begin{tabular}{|l|l|l|l|l|l|l|l|l|l|l|l|l|l|l|l|l|l|l|l|l|}
\hline G & I & V & E & Q & C & C & T & S & I & C & S & L & Y & Q & L & E & N & Y & C & N \\
\hline 10 & 22 & 19 & 19 & 20 & 14 & 14 & 17 & 14 & 22 & 14 & 14 & 22 & 24 & 20 & 22 & 19 & 17 & 24 & 14 & 17 \\
\hline 1 & 2 & 3 & 4 & 5 & 6 & 7 & 8 & 9 & 10 & 11 & 12 & 13 & 14 & 15 & 16 & 17 & 18 & 19 & 20 & 21 \\
\hline
\end{tabular}

1AI0:A

1AI0:B

\begin{tabular}{|l|l|l|l|l|l|l|l|l|l|l|l|l|l|l|}
\hline F & V & N & Q & H & I & C & G & S & H & L & V & E & A & L \\
\hline 23 & 19 & 17 & 20 & 20 & 22 & 14 & 10 & 14 & 20 & 22 & 19 & 19 & 13 & 22 \\
\hline 22 & 23 & 24 & 25 & 26 & 27 & 28 & 29 & 30 & 31 & 32 & 33 & 34 & 35 & 36 \\
\hline
\end{tabular}

\begin{tabular}{|l|l|l|l|l|l|l|l|l|l|l|l|l|l|l|}
\hline Y & L & V & C & G & E & R & G & F & I & Y & T & P & K & T \\
\hline 24 & 22 & 19 & 14 & 10 & 19 & 26 & 10 & 23 & 22 & 24 & 17 & 17 & 24 & 17 \\
\hline 37 & 38 & 39 & 40 & 41 & 42 & 43 & 44 & 45 & 46 & 47 & 48 & 49 & 50 & 51 \\
\hline
\end{tabular}

1AI0:K

\begin{tabular}{|l|l|l|l|l|l|l|l|l|l|l|l|l|l|l|l|l|l|l|l|l|}
\hline G & I & V & E & Q & C & C & T & S & I & C & S & L & Y & Q & L & E & N & Y & C & N \\
\hline 10 & 22 & 19 & 19 & 20 & 14 & 14 & 17 & 14 & 22 & 14 & 14 & 22 & 24 & 20 & 22 & 19 & 17 & 24 & 14 & 17 \\
\hline 256 & 257 & 258 & 259 & 260 & 261 & 262 & 263 & 264 & 265 & 266 & 267 & 268 & 269 & 270 & 271 & 272 & 273 & 274 & 275 & 276 \\
\hline
\end{tabular}

1AI0:L

\begin{tabular}{|l|l|l|l|l|l|l|l|l|l|l|l|l|l|l|}
\hline F & V & N & Q & H & I & C & G & S & H & L & V & E & A & L \\
\hline 23 & 19 & 17 & 20 & 20 & 22 & 14 & 10 & 14 & 20 & 22 & 19 & 19 & 13 & 22 \\
\hline 277 & 278 & 279 & 280 & 281 & 282 & 283 & 284 & 285 & 286 & 287 & 288 & 289 & 290 & 291 \\
\hline
\end{tabular}

\begin{tabular}{|l|l|l|l|l|l|l|l|l|l|l|l|l|l|l|}
\hline Y & L & V & C & G & E & R & G & F & I & Y & T & P & K & T \\
\hline 24 & 22 & 19 & 14 & 10 & 19 & 26 & 10 & 23 & 22 & 24 & 17 & 17 & 24 & 17 \\
\hline 292 & 293 & 294 & 295 & 296 & 297 & 298 & 299 & 300 & 301 & 302 & 303 & 304 & 305 & 306 \\
\hline
\end{tabular}

Number of atoms $=1880$

RANK $=15.657$

\section{Groups C,D,I,]}

1AI0:C

\begin{tabular}{|l|l|l|l|l|l|l|l|l|l|l|l|l|l|l|l|l|l|l|l|l|}
\hline G & I & V & E & Q & C & C & T & S & I & C & S & L & Y & Q & L & E & N & Y & C & N \\
\hline 10 & 22 & 19 & 19 & 20 & 14 & 14 & 17 & 14 & 22 & 14 & 14 & 22 & 24 & 20 & 22 & 19 & 17 & 24 & 14 & 17 \\
\hline 52 & 53 & 54 & 55 & 56 & 57 & 58 & 59 & 60 & 61 & 62 & 63 & 64 & 65 & 66 & 67 & 68 & 69 & 70 & 71 & 72 \\
\hline
\end{tabular}

1AI0:D

\begin{tabular}{|l|l|l|l|l|l|l|l|l|l|l|l|l|l|l|}
\hline F & V & N & Q & H & I & C & G & S & H & L & V & E & A & L \\
\hline 23 & 19 & 17 & 20 & 20 & 22 & 14 & 10 & 14 & 20 & 22 & 19 & 19 & 13 & 22 \\
\hline 73 & 74 & 75 & 76 & 77 & 78 & 79 & 80 & 81 & 82 & 83 & 84 & 85 & 86 & 87 \\
\hline
\end{tabular}




\begin{tabular}{|l|l|l|l|l|l|l|l|l|l|l|l|l|l|l|}
\hline $\mathrm{Y}$ & $\mathrm{L}$ & $\mathrm{V}$ & $\mathrm{C}$ & $\mathrm{G}$ & $\mathrm{E}$ & $\mathrm{R}$ & $\mathrm{G}$ & $\mathrm{F}$ & $\mathrm{I}$ & $\mathrm{Y}$ & $\mathrm{T}$ & $\mathrm{P}$ & $\mathrm{K}$ & $\mathrm{T}$ \\
\hline 24 & 22 & 19 & 14 & 10 & 19 & 26 & 10 & 23 & 22 & 24 & 17 & 17 & 24 & 17 \\
\hline 88 & 89 & 90 & 91 & 92 & 93 & 94 & 95 & 96 & 97 & 98 & 99 & 100 & 101 & 102 \\
\hline
\end{tabular}

1AI0:I

\begin{tabular}{|l|l|l|l|l|l|l|l|l|l|l|l|l|l|l|l|l|l|l|l|l|}
\hline G & I & V & E & Q & C & C & T & S & I & C & S & L & Y & Q & L & E & N & Y & C & N \\
\hline 10 & 22 & 19 & 19 & 20 & 14 & 14 & 17 & 14 & 22 & 14 & 14 & 22 & 24 & 20 & 22 & 19 & 17 & 24 & 14 & 17 \\
\hline 205 & 206 & 207 & 208 & 209 & 210 & 211 & 212 & 213 & 214 & 215 & 216 & 217 & 218 & 219 & 220 & 221 & 222 & 223 & 224 & 225 \\
\hline
\end{tabular}

1AI0:J

\begin{tabular}{|c|c|c|c|c|c|c|c|c|c|c|c|c|c|c|}
\hline$F$ & $\mathrm{~V}$ & $\mathrm{~N}$ & Q & $\mathrm{H}$ & I & C & $\mathrm{G}$ & $S$ & $\mathrm{H}$ & $\mathrm{L}$ & $\mathrm{V}$ & $\mathrm{E}$ & A & $\mathrm{L}$ \\
\hline 23 & 19 & 17 & 20 & 20 & 2 & 14 & 10 & 14 & 20 & 22 & 19 & 19 & 13 & 22 \\
\hline 226 & 227 & 228 & 229 & 230 & 231 & 232 & 233 & 234 & 235 & 236 & 237 & 238 & 239 & 240 \\
\hline Y & $\mathrm{L}$ & V & C & G & $\mathrm{F}$ & $\mathrm{R}$ & $\mathrm{G}$ & $\mathrm{F}$ & & $\mathrm{Y}$ & $\mathrm{T}$ & P & K & $\mathrm{T}$ \\
\hline 24 & 22 & 1 & 14 & 1 & 1 & 2 & 10 & 23 & 2 & 24 & 17 & 17 & 24 & 17 \\
\hline 241 & 242 & 243 & 244 & 245 & 246 & 247 & 248 & 249 & 250 & 251 & 252 & 253 & 254 & 255 \\
\hline
\end{tabular}

Number of atoms $=1880 ;$ RANK $=15.657$

\section{Groups E,F,G,H}

1AI0:E

\begin{tabular}{|l|l|l|l|l|l|l|l|l|l|l|l|l|l|l|l|l|l|l|l|l|}
\hline G & I & V & E & Q & C & C & T & S & I & C & S & L & Y & Q & L & E & N & Y & C & N \\
\hline 10 & 22 & 19 & 19 & 20 & 14 & 14 & 17 & 14 & 22 & 14 & 14 & 22 & 24 & 20 & 22 & 19 & 17 & 24 & 14 & 17 \\
\hline 103 & 104 & 105 & 106 & 107 & 108 & 109 & 110 & 111 & 112 & 113 & 114 & 115 & 116 & 117 & 118 & 119 & 120 & 121 & 122 & 123 \\
\hline
\end{tabular}

1AI0:F

\begin{tabular}{|l|l|l|l|l|l|l|l|l|l|l|l|l|l|l|}
\hline $\mathrm{F}$ & $\mathrm{V}$ & $\mathrm{N}$ & $\mathrm{Q}$ & $\mathrm{H}$ & $\mathrm{I}$ & $\mathrm{C}$ & $\mathrm{G}$ & $\mathrm{S}$ & $\mathrm{H}$ & $\mathrm{L}$ & $\mathrm{V}$ & $\mathrm{E}$ & $\mathrm{A}$ & $\mathrm{L}$ \\
\hline 23 & 19 & 17 & 20 & 20 & 22 & 14 & 10 & 14 & 20 & 22 & 19 & 19 & 13 & 22 \\
\hline 124 & 125 & 126 & 127 & 128 & 129 & 130 & 131 & 132 & 133 & 134 & 135 & 136 & 137 & 138 \\
\hline
\end{tabular}

\begin{tabular}{|l|l|l|l|l|l|l|l|l|l|l|l|l|l|l|}
\hline $\mathrm{Y}$ & $\mathrm{L}$ & $\mathrm{V}$ & $\mathrm{C}$ & $\mathrm{G}$ & $\mathrm{E}$ & $\mathrm{R}$ & $\mathrm{G}$ & $\mathrm{F}$ & $\mathrm{I}$ & $\mathrm{Y}$ & $\mathrm{T}$ & $\mathrm{P}$ & $\mathrm{K}$ & $\mathrm{T}$ \\
\hline 24 & 22 & 19 & 14 & 10 & 19 & 26 & 10 & 23 & 22 & 24 & 17 & 17 & 24 & 17 \\
\hline 139 & 140 & 141 & 142 & 143 & 144 & 145 & 146 & 147 & 148 & 149 & 150 & 151 & 152 & 153 \\
\hline
\end{tabular}

1AI0:G

\begin{tabular}{|l|l|l|l|l|l|l|l|l|l|l|l|l|l|l|l|l|l|l|l|l|}
\hline G & I & V & E & Q & C & C & T & S & I & C & S & L & Y & Q & L & E & N & Y & C & N \\
\hline 10 & 22 & 19 & 19 & 20 & 14 & 14 & 17 & 14 & 22 & 14 & 14 & 22 & 24 & 20 & 22 & 19 & 17 & 24 & 14 & 17 \\
\hline 154 & 155 & 156 & 157 & 158 & 159 & 160 & 161 & 162 & 163 & 164 & 165 & 166 & 167 & 168 & 169 & 170 & 171 & 172 & 173 & 174 \\
\hline
\end{tabular}
$1 \mathrm{AI} 0: \mathrm{H}$

\begin{tabular}{|l|l|l|l|l|l|l|l|l|l|l|l|l|l|l|}
\hline F & V & N & Q & H & I & C & G & S & H & L & V & E & A & L \\
\hline 23 & 19 & 17 & 20 & 20 & 22 & 14 & 10 & 14 & 20 & 22 & 19 & 19 & 13 & 22 \\
\hline 175 & 176 & 177 & 178 & 179 & 180 & 181 & 182 & 183 & 184 & 185 & 186 & 187 & 188 & 183 \\
\hline
\end{tabular}

\begin{tabular}{|l|l|l|l|l|l|l|l|l|l|l|l|l|l|l|}
\hline Y & L & V & C & G & E & R & G & F & I & Y & T & P & K & T \\
\hline 24 & 22 & 19 & 14 & 10 & 19 & 26 & 10 & 23 & 22 & 24 & 17 & 17 & 24 & 17 \\
\hline 190 & 191 & 192 & 193 & 194 & 195 & 196 & 197 & 198 & 199 & 200 & 201 & 202 & 203 & 204 \\
\hline
\end{tabular}

Figure 7 Group of chains E,F,G,H.

Notes: Aforementioned amino acids are positioned from number I to 306. Numbers I, 2, 3, n... present the position of a certain amino acid. This positioning is of key importance for understanding of programmatic, cybernetic and information principles in this protein. The scientific key for interpretation of biochemical processes is the same for insulin and as well as for the other proteins and other sequences in biochemistry. 
Table 2 Overview of the mathematical balance between the positive and negative values of the biocodes of the insulin A chain. Schematic representation of biocode processing (2)

\begin{tabular}{|c|c|c|c|}
\hline $\begin{array}{l}\text { Biocodes } \\
1,2,3, n\end{array}$ & $\begin{array}{l}\text { Biocodes } \\
1,2,3, n\end{array}$ & $(I-2)(+)$ & $(I-2)(-)$ \\
\hline 1 & 2 & 3 & 4 \\
\hline 102219 & 192014 & - & Bio I $=(-) 89795$ \\
\hline 192014 & 141714 & Bio $2=(+) 50300$ & - \\
\hline $1417 \mid 4$ & 221414 & - & Bio $3=(-) 79700$ \\
\hline 221414 & 222420 & - & Bio $4=(-) 1006$ \\
\hline 222420 & 221917 & Bio $5=(+) 503$ & - \\
\hline 221917 & 241417 & - & Bio $6=(-) 19500$ \\
\hline 241417 & 102219 & Bio $7=(+) 139198$ & - \\
\hline SUM & & $(+)|9000|$ & $(-)|9000|$ \\
\hline
\end{tabular}

Number of atoms $=1880 ;$ RANK $=15.657$

\begin{tabular}{|l|l|l|l|}
\hline & A,B,K,L & C,D,I,J & E,F,G,H \\
\hline $\begin{array}{l}\text { Number } \\
\text { of atoms }\end{array}$ & 1880 & 1880 & 1880 \\
\hline Rank & 15657 & 15657 & 15657 \\
\hline Sum & 17537 & 17537 & 17537 \\
\hline
\end{tabular}

Figure 9 Groups of chains $(A, B, K, L),(C, D, I, J)$ and $(E, F, G, H)$

Notes: Each of these three unions of chains has an identical number of amino acids, identical number of atoms and identical sum of position numbers for the said amino acids.

Figure 8 shows the atomic structure 1A10.

Each of these three groups of chains has an identical number of amino acids, an identical number of atoms, and an identical sum of position numbers for these amino acids.

\section{Code 6}

The insulin matrix code can be developed into a periodic system in which each of 20 natural amino acids has an exactly

Table 3 mathematical balance between positive and negative values of analog codes of insulin chain A. Schematic representation of biocode processing (3)

\begin{tabular}{|c|c|c|c|}
\hline $\begin{array}{l}\text { Analog bio } \\
\text { impulse } 1,2,3, \mathrm{n}\end{array}$ & $\begin{array}{l}\text { Analog bio } \\
\text { impulse I,2,3,n }\end{array}$ & $(1-2)(+)$ & $(1-2)(-)$ \\
\hline I & 2 & 3 & 4 \\
\hline 912201 & 410291 & $\begin{array}{l}\text { Analog bio } \\
\text { (+) } 501910\end{array}$ & - \\
\hline 410291 & $4|7| 4 \mid$ & - & $\begin{array}{l}\text { Analog bio }(-) \\
6850\end{array}$ \\
\hline $4|7| 4 \mid$ & $4|4| 22$ & $\begin{array}{l}\text { Analog bio } \\
\text { (+) } 3019\end{array}$ & - \\
\hline $4|4| 22$ & 24222 & $\begin{array}{l}\text { Analog bio } \\
(+) 389900\end{array}$ & - \\
\hline 24222 & 719122 & - & $\begin{array}{l}\text { Analog bio (-) } \\
694900\end{array}$ \\
\hline 719122 & $7|4| 42$ & $\begin{array}{l}\text { Analog bio } \\
(+) 4980\end{array}$ & - \\
\hline $7|4| 42$ & 912201 & - & $\begin{array}{l}\text { Analog bio }(-) \\
198059\end{array}$ \\
\hline SUM & & (+) 899809 & (-) 899809 \\
\hline
\end{tabular}

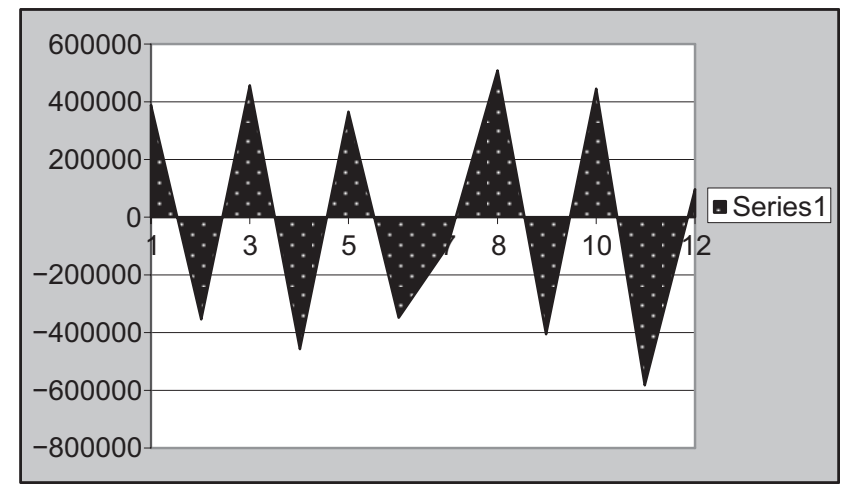

Figure $8 \mathrm{~A}$ schematic diagram to show the positive and negative output biocodes.

established place. On the basis of this system, amino acids are distributed according to the criteria of mathematical similarity or difference (Figure 10).

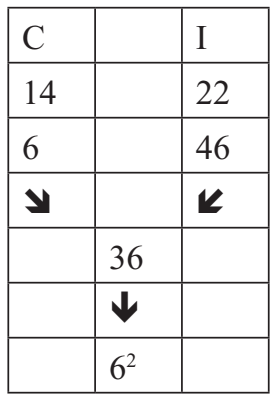

\begin{tabular}{|l|l|l|}
\hline I & & C \\
\hline 22 & & 14 \\
\hline 2 & & 40 \\
\hline $\boldsymbol{y}$ & & $\boldsymbol{K}$ \\
\hline & 36 & \\
\hline & $\boldsymbol{V}$ & \\
\hline & $6^{2}$ & \\
\hline
\end{tabular}

\begin{tabular}{|l|l|l|}
\hline S & & L \\
\hline 14 & & 22 \\
\hline 30 & & 38 \\
\hline $\boldsymbol{y}$ & & $\boldsymbol{K}$ \\
\hline & 36 & \\
\hline & $\boldsymbol{V}$ & \\
\hline & $6^{2}$ & \\
\hline
\end{tabular}

\begin{tabular}{|l|l|l|}
\hline $\mathrm{I}$ & & $\mathrm{C}$ \\
\hline 22 & & 14 \\
\hline 10 & & 7 \\
\hline $\boldsymbol{y}$ & & $\boldsymbol{K}$ \\
\hline & 36 & \\
\hline & $\boldsymbol{V}$ & \\
\hline & $6^{2}$ & \\
\hline
\end{tabular}

\begin{tabular}{|l|l|l|}
\hline $\mathrm{C}$ & & $\mathrm{L}$ \\
\hline 14 & & 22 \\
\hline 28 & & 36 \\
\hline $\boldsymbol{y}$ & & $\boldsymbol{K}$ \\
\hline & 36 & \\
\hline & $\boldsymbol{\downarrow}$ & \\
\hline & $6^{2}$ & \\
\hline
\end{tabular}

\begin{tabular}{|l|l|l|}
\hline $\mathrm{L}$ & & $\mathrm{S}$ \\
\hline 22 & & 14 \\
\hline 13 & & 9 \\
\hline $\boldsymbol{y}$ & & $\boldsymbol{K}$ \\
\hline & 36 & \\
\hline & $\boldsymbol{V}$ & \\
\hline & $6^{2}$ & \\
\hline
\end{tabular}

Figure 10 Distribution of amino acids according to the criteria of mathematical similarity or difference.

Notes: The sum of the atoms in these amino acids, we, as a result, are given the number 36 , or $6^{2}$. That was the code that represents one of the quantitative characteristics of the given genetic information.

In this example, the quantitative characteristics of genetic information are given in the sign of the code 6 . That code links the corresponding groups of amino acids. 


\section{Rank of the biocodes}

Some groups of amino acids that are discussed here are connected by the discrete code 11 . Here are some examples (Figure 11)

Fragment 1

\begin{tabular}{|l|l|l|}
\hline I & & S \\
\hline 22 & & 14 \\
\hline 10 & & 12 \\
\hline $\mathbf{y}$ & & $\mathbf{K}$ \\
\hline & 22 & \\
\hline $\mathbf{K}$ & & $\mathbf{Y}$ \\
\hline 11 & & 11 \\
\hline
\end{tabular}

$(10+12)=22 ; 22=(11+11)$

Fragment 2

\begin{tabular}{|l|l|l|}
\hline S & & L \\
\hline 14 & & 22 \\
\hline 9 & & 13 \\
\hline $\boldsymbol{y}$ & & $\mathbf{K}$ \\
\hline & 22 & \\
\hline $\mathbf{K}$ & & $\mathbf{y}$ \\
\hline 11 & & 11 \\
\hline
\end{tabular}

Fragment 3

\begin{tabular}{|l|l|l|}
\hline $\mathrm{C}$ & & $\mathrm{L}$ \\
\hline 14 & & 22 \\
\hline 6 & & 16 \\
\hline $\boldsymbol{y}$ & & $\mathbf{K}$ \\
\hline & 22 & \\
\hline $\mathbf{K}$ & & $\mathbf{Y}$ \\
\hline 11 & & 11 \\
\hline
\end{tabular}

etc.

Figure II Groups of amino acids connected by the discrete code II. Notes: In the aforementioned examples, amino acids are connected by the code 6 , and their position numbers are connected with code II. As it can be observed, quantitative characteristics of the biochemistry of insulin apply to the position number.

In the aforementioned examples, amino acids are connected by the code 6 , and their position numbers are connected with code 11 . As can be seen, the quantitative characteristics of the biochemistry of insulin apply to the position number.

\section{Biocode I I}

The aforementioned code 11 also connects groups of amino acids. We can see that in the following example (Figure 12).

\begin{tabular}{|c|c|c|c|c|c|c|c|c|c|c|}
\hline $\mathrm{G}$ & $\mathrm{V}$ & Q & $\mathrm{C}$ & $\mathrm{T}$ & $\mathrm{C}$ & $Y$ & $Q$ & $\mathrm{E}$ & $Y$ & $\mathrm{~N}$ \\
\hline 10 & 19 & 20 & 14 & 17 & 14 & 24 & 20 & 19 & 24 & 17 \\
\hline \multicolumn{11}{|c|}{$\downarrow$} \\
\hline \multicolumn{11}{|c|}{$\begin{array}{l}1019201417142420192417 \\
\downarrow\end{array}$} \\
\hline
\end{tabular}

We will give a few more examples of coding of amino acids of insulin with code 11: (Figure 13-15).

Biocode $11(1)$
\begin{tabular}{|l|l|l|}
\hline $\mathrm{G}$ & & $\mathrm{N}$ \\
\hline 10 & & 17 \\
\hline 1 & & 21 \\
\hline $\boldsymbol{y}$ & & $\boldsymbol{K}$ \\
\hline & 121 & \\
\hline & $\boldsymbol{V}$ & \\
\hline & $11^{2}$ & \\
\hline
\end{tabular}

Figure 13 Connection of the rank of amino acids $\mathrm{G}$ and $\mathrm{N}$. Notes: In this example we connected the position number of amino acids $\mathrm{G}$ and $\mathrm{N}$, and got number 121 as result.

In this example we connected the position number of amino acids $\mathrm{G}$ and $\mathrm{N}$, and got number 121 or 112 as result.

Biocode 11(2)

\begin{tabular}{|l|l|l|}
\hline $\mathrm{V}$ & & $\mathrm{Y}$ \\
\hline 19 & & 24 \\
\hline 3 & & 19 \\
\hline $\mathbf{y}$ & & $\boldsymbol{K}$ \\
\hline & 319 & \\
\hline & $\boldsymbol{\downarrow}$ & \\
\hline & $(11 \times \mathrm{Y})$ & \\
\hline
\end{tabular}

Figure 14 Connection of the rank of amino acids $\mathrm{V}$ and $\mathrm{Y}$.

Notes: In this example we connected the position number of amino acids $\mathrm{V}$ and $\mathrm{Y}$, and got number $(\mathrm{I} \mathrm{I} \times \mathrm{Y})$ as result.

\section{Biocode 11(3)}

\begin{tabular}{|l|l|l|}
\hline $\mathrm{Q}$ & & $\mathrm{E}$ \\
\hline 20 & & 19 \\
\hline 5 & & 17 \\
\hline $\boldsymbol{y}$ & & $\boldsymbol{K}$ \\
\hline & 517 & \\
\hline & $\boldsymbol{V}$ & \\
\hline & $(11 \times \mathrm{Y} 1)$ & \\
\hline
\end{tabular}

Figure 15 Connection of the rank of amino acids $\mathrm{Q}$ and $\mathrm{E}$. Notes: In this example we connected the position number of amino acids $Q$ and $E$, and got number $(\mathrm{I} \mid \times \mathrm{YI})$ as result.

\begin{tabular}{|l|l|l|l|l|l|l|l|l|l|l|l|l|}
\hline G & V & Q & C & T & C & Y & Q & E & Y & N & SUM & Code \\
\hline 10 & 19 & 20 & 14 & 17 & 14 & 24 & 20 & 19 & 24 & 17 & 198 & $(198: X)=11:$ \\
\hline 1 & 3 & 5 & 7 & 8 & 11 & 14 & 15 & 17 & 19 & 21 & 121 & $(121: 11)=11:$ \\
\hline- & - & - & - & - & - & - & - & - & - & - & 319 & $(319: Y)=11 ;$ \\
\hline
\end{tabular}

Figure 12 Discrete code II connects groups of amino acids.

Notes: These groups of amino acids are connected by the discrete code II. 


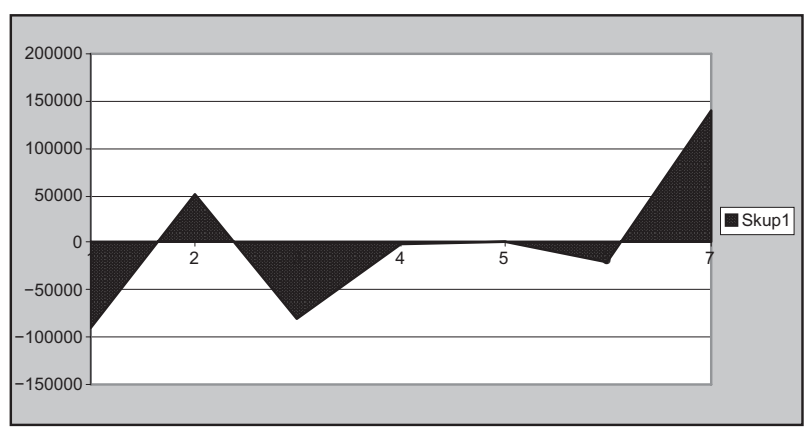

Figure 16 A schematic diagram to show the positive and negative output biocodes of the insulin A chain.

From the previous examples it can be seen that this protein has quantitative characteristics. It can be concluded that there is a connection between these quantitative characteristics in the process of transfer of genetic information and the qualitative appearance of the given genetic processes.

\section{Biofrequency r6 insulin hexamer (d ai02)}

Insulin is composed of amino acids with various numeric values. These numeric values are in an irregular order. For example, the first one has 10 atoms, the second one 22 .

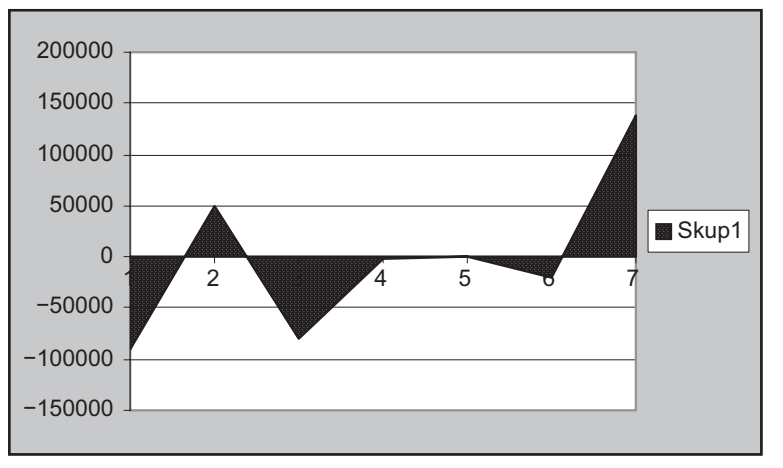

Figure 17 A schematic diagram to show the positive and negative output analog biocodes of the insulin $\mathrm{C}$ chain.

Their frequency is X. The second amino acid has 22 atoms, and the third one 19. Their frequency is Y, etc. Frequency is the measurement for establishment of intervals of numeric values of amino acids in proteins. This value can be positive, negative, or zero. These frequencies demonstrate a completely new dimension of protein sequencing. Using these frequencies we can establish which of the amino acids are of primary and which are of secondary significance in the biochemical processes of insulin. A practical example:

\begin{tabular}{|l|l|l|l|l|l|l|l|l|l|l|l|l|l|l|l|l|l|l|l|l|}
\hline $\mathrm{G}$ & $\mathrm{I}$ & $\mathrm{V}$ & $\mathrm{E}$ & $\mathrm{Q}$ & $\mathrm{C}$ & $\mathrm{C}$ & $\mathrm{T}$ & $\mathrm{S}$ & $\mathrm{I}$ & $\mathrm{C}$ & $\mathrm{S}$ & $\mathrm{L}$ & $\mathrm{Y}$ & $\mathrm{Q}$ & $\mathrm{L}$ & $\mathrm{E}$ & $\mathrm{N}$ & $\mathrm{Y}$ & $\mathrm{C}$ & $\mathrm{N}$ \\
\hline 10 & 22 & 19 & 19 & 20 & 14 & 14 & 17 & 14 & 22 & 14 & 14 & 22 & 24 & 20 & 22 & 19 & 17 & 24 & 14 & 17 \\
\hline 1 & 2 & 3 & 4 & 5 & 6 & 7 & 8 & 9 & 10 & 11 & 12 & 13 & 14 & 15 & 16 & 17 & 18 & 19 & 20 & 21 \\
\hline 10 & 12 & -3 & 0 & 1 & -6 & 0 & 3 & -3 & 8 & -8 & 0 & 8 & 2 & -4 & 2 & -3 & -2 & 7 & -10 & 3 \\
\hline
\end{tabular}

From 0 to $10=10$; from 10 to $22=12$; from 22 to $19=(-) 3$; from 19 to $10=0$, etc.

A schematic representation of the amino acids and their frequency is shown in Figure 17.

\begin{tabular}{|l|l|l|l|l|l|l|l|l|l|l|l|l|l|l|}
\hline F & V & N & Q & H & I & C & G & S & H & L & V & E & A & L \\
\hline 23 & 19 & 17 & 20 & 20 & 22 & 14 & 10 & 14 & 20 & 22 & 19 & 19 & 13 & 22 \\
\hline 22 & 23 & 24 & 25 & 26 & 27 & 28 & 29 & 30 & 31 & 32 & 33 & 34 & 35 & 36 \\
\hline 6 & -4 & -2 & 3 & 0 & 2 & -8 & -4 & 4 & 6 & 2 & -3 & 0 & -6 & 9 \\
\hline
\end{tabular}

\begin{tabular}{|l|l|l|l|l|l|l|l|l|l|l|l|l|l|l|}
\hline Y & L & V & C & G & E & R & G & F & I & Y & T & P & K & T \\
\hline 24 & 22 & 19 & 14 & 10 & 19 & 26 & 10 & 23 & 22 & 24 & 17 & 17 & 24 & 17 \\
\hline 37 & 38 & 39 & 40 & 41 & 42 & 43 & 44 & 45 & 46 & 47 & 48 & 49 & 50 & 51 \\
\hline 2 & -2 & -3 & -5 & -4 & 9 & 7 & -16 & 13 & -1 & 2 & -7 & 0 & 7 & -7 \\
\hline
\end{tabular}

\begin{tabular}{|l|l|l|l|l|l|l|l|l|l|l|l|l|l|l|l|l|l|l|l|l|}
\hline G & I & V & E & Q & C & C & T & S & I & C & S & L & Y & Q & L & E & N & Y & C & N \\
\hline 10 & 22 & 19 & 19 & 20 & 14 & 14 & 17 & 14 & 22 & 14 & 14 & 22 & 24 & 20 & 22 & 19 & 17 & 24 & 14 & 17 \\
\hline 52 & 53 & 54 & 55 & 56 & 57 & 58 & 59 & 60 & 61 & 62 & 63 & 64 & 65 & 66 & 67 & 68 & 69 & 70 & 71 & 72 \\
\hline-7 & 12 & -3 & 0 & 1 & -6 & 0 & 3 & -3 & 8 & -8 & 0 & 8 & 2 & -4 & 2 & -3 & -2 & 7 & -10 & 3 \\
\hline
\end{tabular}




\begin{tabular}{|l|l|l|l|l|l|l|l|l|l|l|l|l|l|l|}
\hline F & V & N & Q & H & I & C & G & S & H & L & V & E & A & L \\
\hline 23 & 19 & 17 & 20 & 20 & 22 & 14 & 10 & 14 & 20 & 22 & 19 & 19 & 13 & 22 \\
\hline 73 & 74 & 75 & 76 & 77 & 78 & 79 & 80 & 81 & 82 & 83 & 84 & 85 & 86 & 87 \\
\hline 6 & -4 & -2 & 3 & 0 & 2 & -8 & -4 & 4 & 6 & 2 & -3 & 0 & -6 & 9 \\
\hline
\end{tabular}

\begin{tabular}{|l|l|l|l|l|l|l|l|l|l|l|l|l|l|l|}
\hline Y & L & V & C & G & E & R & G & F & I & Y & T & P & K & T \\
\hline 24 & 22 & 19 & 14 & 10 & 19 & 26 & 10 & 23 & 22 & 24 & 17 & 17 & 24 & 17 \\
\hline 88 & 89 & 90 & 91 & 92 & 93 & 94 & 95 & 96 & 97 & 98 & 99 & 100 & 101 & 102 \\
\hline 2 & -2 & -3 & -5 & -4 & 9 & 7 & -16 & 13 & -1 & 2 & -7 & 0 & 7 & -7 \\
\hline
\end{tabular}

\begin{tabular}{|l|l|l|l|l|l|l|l|l|l|l|l|l|l|l|l|l|l|l|l|l|}
\hline $\mathrm{G}$ & $\mathrm{I}$ & $\mathrm{V}$ & $\mathrm{E}$ & $\mathrm{Q}$ & $\mathrm{C}$ & $\mathrm{C}$ & $\mathrm{T}$ & $\mathrm{S}$ & $\mathrm{I}$ & $\mathrm{C}$ & $\mathrm{S}$ & $\mathrm{L}$ & $\mathrm{Y}$ & $\mathrm{Q}$ & $\mathrm{L}$ & $\mathrm{E}$ & $\mathrm{N}$ & $\mathrm{Y}$ & $\mathrm{C}$ & $\mathrm{N}$ \\
\hline 10 & 22 & 19 & 19 & 20 & 14 & 14 & 17 & 14 & 22 & 14 & 14 & 22 & 24 & 20 & 22 & 19 & 17 & 24 & 14 & 17 \\
\hline 103 & 104 & 105 & 106 & 107 & 108 & 109 & 110 & 111 & 112 & 113 & 114 & 115 & 116 & 117 & 118 & 119 & 120 & 121 & 122 & 123 \\
\hline-7 & 12 & -3 & 0 & 1 & -6 & 0 & 3 & -3 & 8 & -8 & 0 & 8 & 2 & -4 & 2 & -3 & -2 & 7 & -10 & 3 \\
\hline
\end{tabular}

\begin{tabular}{|l|l|l|l|l|l|l|l|l|l|l|l|l|l|l|}
\hline $\mathrm{F}$ & $\mathrm{V}$ & $\mathrm{N}$ & $\mathrm{Q}$ & $\mathrm{H}$ & $\mathrm{I}$ & $\mathrm{C}$ & $\mathrm{G}$ & $\mathrm{S}$ & $\mathrm{H}$ & $\mathrm{L}$ & $\mathrm{V}$ & $\mathrm{E}$ & $\mathrm{A}$ & $\mathrm{L}$ \\
\hline 23 & 19 & 17 & 20 & 20 & 22 & 14 & 10 & 14 & 20 & 22 & 19 & 19 & 13 & 22 \\
\hline 124 & 125 & 126 & 127 & 128 & 129 & 130 & 131 & 132 & 133 & 134 & 135 & 136 & 137 & 138 \\
\hline 6 & -4 & -2 & 3 & 0 & 2 & -8 & -4 & 4 & 6 & 2 & -3 & 0 & -6 & 9 \\
\hline
\end{tabular}

\begin{tabular}{|l|l|l|l|l|l|l|l|l|l|l|l|l|l|l|}
\hline Y & L & V & C & G & E & R & G & F & I & Y & T & P & K & T \\
\hline 24 & 22 & 19 & 14 & 10 & 19 & 26 & 10 & 23 & 22 & 24 & 17 & 17 & 24 & 17 \\
\hline 139 & 140 & 141 & 142 & 143 & 144 & 145 & 146 & 147 & 148 & 149 & 150 & 151 & 152 & 153 \\
\hline 2 & -2 & -3 & -5 & -4 & 9 & 7 & -16 & 13 & -1 & 2 & -7 & 0 & 7 & -7 \\
\hline
\end{tabular}

\begin{tabular}{|l|l|l|l|l|l|l|l|l|l|l|l|l|l|l|l|l|l|l|l|l|}
\hline $\mathrm{G}$ & $\mathrm{I}$ & $\mathrm{V}$ & $\mathrm{E}$ & $\mathrm{Q}$ & $\mathrm{C}$ & $\mathrm{C}$ & $\mathrm{T}$ & $\mathrm{S}$ & $\mathrm{I}$ & $\mathrm{C}$ & $\mathrm{S}$ & $\mathrm{L}$ & $\mathrm{Y}$ & $\mathrm{Q}$ & $\mathrm{L}$ & $\mathrm{E}$ & $\mathrm{N}$ & $\mathrm{Y}$ & $\mathrm{C}$ & $\mathrm{N}$ \\
\hline 10 & 22 & 19 & 19 & 20 & 14 & 14 & 17 & 14 & 22 & 14 & 14 & 22 & 24 & 20 & 22 & 19 & 17 & 24 & 14 & 17 \\
\hline 154 & 155 & 156 & 157 & 158 & 159 & 160 & 161 & 162 & 163 & 164 & 165 & 166 & 167 & 168 & 169 & 170 & 171 & 172 & 173 & 174 \\
\hline-7 & 12 & -3 & 0 & 1 & -6 & 0 & 3 & -3 & 8 & -8 & 0 & 8 & 2 & -4 & 2 & -3 & -2 & 7 & -10 & 3 \\
\hline
\end{tabular}

\begin{tabular}{|l|l|l|l|l|l|l|l|l|l|l|l|l|l|l|}
\hline F & V & N & Q & H & I & C & G & S & H & L & V & E & A & L \\
\hline 23 & 19 & 17 & 20 & 20 & 22 & 14 & 10 & 14 & 20 & 22 & 19 & 19 & 13 & 22 \\
\hline 175 & 176 & 177 & 178 & 179 & 180 & 181 & 182 & 183 & 184 & 185 & 186 & 187 & 188 & 189 \\
\hline 6 & -4 & -2 & 3 & 0 & 2 & -8 & -4 & 4 & 6 & 2 & -3 & 0 & -6 & 9 \\
\hline
\end{tabular}

\begin{tabular}{|l|l|l|l|l|l|l|l|l|l|l|l|l|l|l|}
\hline $\mathrm{Y}$ & $\mathrm{L}$ & $\mathrm{V}$ & $\mathrm{C}$ & $\mathrm{G}$ & $\mathrm{E}$ & $\mathrm{R}$ & $\mathrm{G}$ & $\mathrm{F}$ & $\mathrm{I}$ & $\mathrm{Y}$ & $\mathrm{T}$ & $\mathrm{P}$ & $\mathrm{K}$ & $\mathrm{T}$ \\
\hline 24 & 22 & 19 & 14 & 10 & 19 & 26 & 10 & 23 & 22 & 24 & 17 & 17 & 24 & 17 \\
\hline 190 & 191 & 192 & 193 & 194 & 195 & 196 & 197 & 198 & 199 & 200 & 201 & 202 & 203 & 204 \\
\hline 2 & -2 & -3 & -5 & -4 & 9 & 7 & -16 & 13 & -1 & 2 & -7 & 0 & 7 & -7 \\
\hline
\end{tabular}

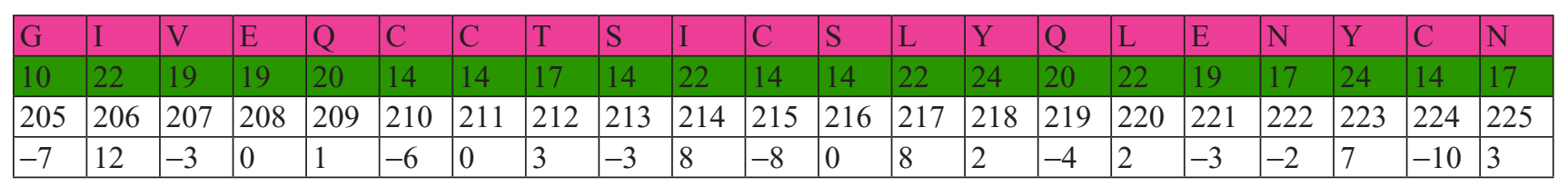




\begin{tabular}{|l|l|l|l|l|l|l|l|l|l|l|l|l|l|l|}
\hline F & V & N & Q & H & I & C & G & S & H & L & V & E & A & L \\
\hline 23 & 19 & 17 & 20 & 20 & 22 & 14 & 10 & 14 & 20 & 22 & 19 & 19 & 13 & 22 \\
\hline 226 & 227 & 228 & 229 & 230 & 231 & 232 & 233 & 234 & 235 & 236 & 237 & 238 & 239 & 240 \\
\hline 6 & -4 & -2 & 3 & 0 & 2 & -8 & -4 & 4 & 6 & 2 & -3 & 0 & -6 & 9 \\
\hline
\end{tabular}

\begin{tabular}{|l|l|l|l|l|l|l|l|l|l|l|l|l|l|l|}
\hline $\mathrm{Y}$ & $\mathrm{L}$ & $\mathrm{V}$ & $\mathrm{C}$ & $\mathrm{G}$ & $\mathrm{E}$ & $\mathrm{R}$ & $\mathrm{G}$ & $\mathrm{F}$ & $\mathrm{I}$ & $\mathrm{Y}$ & $\mathrm{T}$ & $\mathrm{P}$ & $\mathrm{K}$ & $\mathrm{T}$ \\
\hline 24 & 22 & 19 & 14 & 10 & 19 & 26 & 10 & 23 & 22 & 24 & 17 & 17 & 24 & 17 \\
\hline 241 & 242 & 243 & 244 & 245 & 246 & 247 & 248 & 249 & 250 & 251 & 252 & 253 & 254 & 255 \\
\hline 2 & -2 & -3 & -5 & -4 & 9 & 7 & -16 & 13 & -1 & 2 & -7 & 0 & 7 & -7 \\
\hline
\end{tabular}

\begin{tabular}{|l|l|l|l|l|l|l|l|l|l|l|l|l|l|l|l|l|l|l|l|l|}
\hline G & I & V & E & Q & C & C & T & S & I & C & S & L & Y & Q & L & E & N & Y & C & N \\
\hline 10 & 22 & 19 & 19 & 20 & 14 & 14 & 17 & 14 & 22 & 14 & 14 & 22 & 24 & 20 & 22 & 19 & 17 & 24 & 14 & 17 \\
\hline 256 & 257 & 258 & 259 & 260 & 261 & 262 & 263 & 264 & 265 & 266 & 267 & 268 & 269 & 270 & 271 & 272 & 273 & 274 & 275 & 276 \\
\hline-7 & 12 & -3 & 0 & 1 & -6 & 0 & 3 & -3 & 8 & -8 & 0 & 8 & 2 & -4 & 2 & -3 & -2 & 7 & -10 & 3 \\
\hline
\end{tabular}

\begin{tabular}{|l|l|l|l|l|l|l|l|l|l|l|l|l|l|l|}
\hline F & V & N & Q & H & I & C & G & S & H & L & V & E & A & L \\
\hline 23 & 19 & 17 & 20 & 20 & 22 & 14 & 10 & 14 & 20 & 22 & 19 & 19 & 13 & 22 \\
\hline 277 & 278 & 279 & 280 & 281 & 282 & 283 & 284 & 285 & 286 & 287 & 288 & 289 & 290 & 291 \\
\hline 6 & -4 & -2 & 3 & 0 & 2 & -8 & -4 & 4 & 6 & 2 & -3 & 0 & -6 & 9 \\
\hline
\end{tabular}

\begin{tabular}{|l|l|l|l|l|l|l|l|l|l|l|l|l|l|l|l|}
\hline $\mathrm{Y}$ & $\mathrm{L}$ & $\mathrm{V}$ & $\mathrm{C}$ & $\mathrm{G}$ & $\mathrm{E}$ & $\mathrm{R}$ & $\mathrm{G}$ & $\mathrm{F}$ & $\mathrm{I}$ & $\mathrm{Y}$ & $\mathrm{T}$ & $\mathrm{P}$ & $\mathrm{K}$ & $\mathrm{T}$ & \\
\hline 24 & 22 & 19 & 14 & 10 & 19 & 26 & 10 & 23 & 22 & 24 & 17 & 17 & 24 & 17 & \\
\hline 292 & 293 & 294 & 295 & 296 & 297 & 298 & 299 & 300 & 301 & 302 & 303 & 304 & 305 & 306 & \\
\hline 2 & -2 & -3 & -5 & -4 & 9 & 7 & -16 & 13 & -1 & 2 & -7 & 0 & 7 & -7 & -17 \\
\hline
\end{tabular}

Bio frequency $(+)$

\begin{tabular}{|l|l|l|l|l|l|l|l|l|l|l|l|l|l|l|l|l|l|l|}
\hline G & I & Q & T & I & L & Y & L & Y & N & F & Q & I & S & H & L & L & Y & E \\
\hline 10 & 22 & 20 & 17 & 22 & 22 & 24 & 22 & 24 & 17 & 23 & 20 & 22 & 14 & 20 & 22 & 22 & 24 & 19 \\
\hline 1 & 2 & 5 & 8 & 10 & 13 & 14 & 16 & 19 & 21 & 22 & 25 & 27 & 30 & 31 & 32 & 36 & 37 & 42 \\
\hline 10 & 12 & 1 & 3 & 8 & 8 & 2 & 2 & 7 & 3 & 6 & 3 & 2 & 4 & 6 & 2 & 9 & 2 & 9 \\
\hline
\end{tabular}

Bio frequency $(+)=(10+12+1+3 \cdots+7)=(+) 718$

Bio frequency (zero)

\begin{tabular}{|l|l|l|l|l|l|l|l|l|l|l|l|l|l|l|l|l|l|}
\hline E & $\mathrm{C}$ & $\mathrm{S}$ & $\mathrm{H}$ & $\mathrm{E}$ & $\mathrm{P}$ & $\mathrm{E}$ & $\mathrm{C}$ & $\mathrm{S}$ & $\mathrm{H}$ & $\mathrm{E}$ & $\mathrm{P}$ & $\mathrm{E}$ & $\mathrm{C}$ & $\mathrm{S}$ & $\mathrm{H}$ & $\mathrm{E}$ & $\mathrm{P}$ \\
\hline 19 & 14 & 14 & 20 & 19 & 17 & 19 & 14 & 14 & 20 & 19 & 17 & 19 & 14 & 14 & 20 & 19 & 17 \\
\hline 4 & 7 & 12 & 26 & 34 & 49 & 55 & 58 & 63 & 77 & 85 & 100 & 106 & 109 & 114 & 128 & 136 & 151 \\
\hline 0 & 0 & 0 & 0 & 0 & 0 & 0 & 0 & 0 & 0 & 0 & 0 & 0 & 0 & 0 & 0 & 0 & 0 \\
\hline
\end{tabular}

\begin{tabular}{|l|l|l|l|l|l|l|l|l|l|l|l|l|l|l|l|l|l|}
\hline $\mathrm{E}$ & $\mathrm{C}$ & $\mathrm{S}$ & $\mathrm{H}$ & $\mathrm{E}$ & $\mathrm{P}$ & $\mathrm{E}$ & $\mathrm{C}$ & $\mathrm{S}$ & $\mathrm{H}$ & $\mathrm{E}$ & $\mathrm{P}$ & $\mathrm{E}$ & $\mathrm{C}$ & $\mathrm{S}$ & $\mathrm{H}$ & $\mathrm{E}$ & $\mathrm{P}$ \\
\hline 19 & 14 & 14 & 20 & 19 & 17 & 19 & 14 & 14 & 20 & 19 & 17 & 19 & 14 & 14 & 20 & 19 & 17 \\
\hline 157 & 160 & 165 & 179 & 187 & 202 & 208 & 211 & 216 & 230 & 238 & 253 & 259 & 262 & 267 & 281 & 289 & 304 \\
\hline 0 & 0 & 0 & 0 & 0 & 0 & 0 & 0 & 0 & 0 & 0 & 0 & 0 & 0 & 0 & 0 & 0 & 0 \\
\hline
\end{tabular}


Bio frequency (-)

\begin{tabular}{|l|l|l|l|l|l|l|l|l|l|l|l|l|l|l|l|l|}
\hline V & C & S & C & Q & E & N & C & V & N & C & G & V & A & L & V & C \\
\hline 19 & 14 & 14 & 14 & 20 & 19 & 17 & 14 & 19 & 17 & 14 & 10 & 19 & 13 & 22 & 19 & 14 \\
\hline 3 & 6 & 9 & 11 & 15 & 17 & 18 & 20 & 23 & 24 & 28 & 29 & 33 & 35 & 38 & 39 & 40 \\
\hline-3 & -6 & -3 & -8 & -4 & -3 & -2 & -10 & -4 & -2 & -8 & -4 & -3 & -6 & -2 & -3 & -5 \\
\hline
\end{tabular}

Figure 18 Frequency of insulin.

Notes: Insulin is composed of amino acids with various numerical values. These numerical values are in irregular order. For example, the first one has 10 atoms, the second one 22. Their 10, 12, (-)3, 0 etc...... Frequency is the measurement for establishment of intervals of numerical values of amino acids in proteins.

etc.

Bio frequency $(-)=[(-3)+(-6)+(-3) \cdots+(-17)]=(-) 718$

\section{Results}

Bio frequency $=[(-) 718 \leftrightarrow(+) 718]$

Therefore, there is a mathematical balance between the group of amino acids with a positive frequency and those with a negative frequency. Amino acids with a positive frequency have a primary role in the mathematical picture of that protein, and the negative frequencies have a secondary role in it. We assume that amino acids with a positive frequency have a primary role in the biochemical picture of that protein, and the negative frequencies have a secondary role in it. If this really is the case and research on an experimental level proves it, a radically new way of learning about biochemical processes will emerge.

Within insulin's constituents, 133 amino acids have a positive frequency. These amino acids have 2848 atoms. There are 137 amino acids with a negative frequency. These amino acids have 2174 atoms. Number 133 from the union of acids with a positive frequency is the discrete number that we can use for decoding a matrix with positive and negative frequencies. Here are some examples:

Example 1

$[(137+2174)+(133+2848)]=[(133+133)+(718 \times Y)]$

$\mathrm{Y}=7$;

$133=(7+7+7 \cdots,+7)$

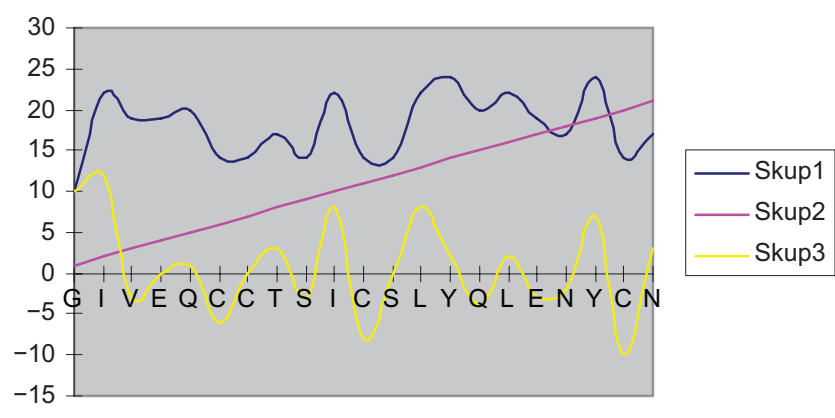

Figure 19 Bio frequency $(+),(-)$, and zero.
Example 2

4712 || 2174

8482 || 2848

$(4712+2174+8482+2848)=[(133+133)+(718 \times Y)]$

$\mathrm{Y}=25$

\section{Levels of frequencies}

In the group of amino acids with a positive frequency there are two subunions. One is the subunion with positive frequencies, and the other one is a subunion with negative frequencies. Also, in the union of amino acids with negative frequency, there are subunions with positive and negative frequencies. These groups also have their subunions with positive and negative frequencies. These groups demonstrate a new dimension of the biochemistry of insulin. We expect that this discovery will unlock many of the secrets of protein biochemistry in the future.

\section{Discussion}

The results of our research show that the process of sequencing molecules is conditioned and arranged not only according to chemical and biochemical laws, but also according to program, cybernetic, and informational laws. At the first stage of our research we replaced nucleotides from the amino acid code matrix with the numbers of atoms in those nucleotides. Translation of the biochemical language of these amino acids into a digital language may be very useful for developing new methods of predicting protein subcellular localization, membrane protein type, secondary protein structure prediction, or indeed any other protein attribute. Since the concept of Chou's pseudo amino acid composition was proposed, ${ }^{1,2}$ there have been many attempts to use various digital numbers to represent the 20 native amino acids in order to reflect better the sequence-order effects involved. Some investigators have used complexity measure factor, ${ }^{3}$ whereas others have used values derived from cellular automata, ${ }^{4-7}$ hydrophobic and/or hydrophilic values, ${ }^{8-16}$ Fourier transform techniques, ${ }^{17,18}$ or physicochemical distance. ${ }^{19}$ 
It is going to be possible to use a completely new strategy of research in genetics in the future. However, close investigation of all these relationships, which are the outcomes of periodic laws (more specifically the law of binary coding) is necessary to be able to decode the conformational, stereochemical, and digital structure of proteins.

\section{Disclosure}

The author reports no conflict of interest in this research.

\section{References}

1. Chou KC. A new branch of proteomics: Prediction of protein cellular attributes. In: Weinrer PW, Lu Q, editors. Gene Cloning and Expression Technologies. Westborough, MA: Eaton Publishing; 2002.

2. Chou KC. Prediction of protein cellular attributes using pseudo amino acid composition. Proteins. 2001;43:246-255.

3. Xiao X, Shao S, Ding Y, Huang Z, Huang Y, Chou KC. Using complexity measure factor to predict protein subcellular location. Amino Acids. 2005;28:57-61.

4. Xiao X, Shao S, Ding Y, Huang Z, Chen X, Chou CK. Using cellular automata to generate image representation for biological sequences. Amino Acids. 2005;28:29-35.

5. Xiao X, Shao S, Ding Y, Huang Z, Chen X, Chou KC. An application of gene comparative image for predicting the effect on replication ratio by HBV virus gene missense mutation. J Theor Biol. 2005;235: 555-565.

6. Xiao X, Shao SH, Huang ZD, Chou KC. Using pseudo amino acid composition to predict protein structural classes: Approached with complexity measure factor. J Comput Chem. 2006;27:478-482.

7. Xiao X, Shao SH, Ding YS, Huang ZD, Chou KD. Using cellular automata images and pseudo amino acid composition to predict protein sub-cellular location. Amino Acids. 2006;30:49-54.

8. Chou KC. Using amphiphilic pseudo amino acid composition to predict enzyme subfamily classes. Bioinformatics. 2005;21:10-9.

9. Chou KC, Cai YD. Prediction of membrane protein types by incorporating amphipathic effects. J Chem Inf Model. 2005;45:407-413.

10. Feng ZP. Prediction of the subcellular location of prokaryotic proteins based on a new representation of the amino acid composition. Biopolymers. 2001;58:491-499.

11. Feng ZP. An overview on predicting the subcellular location of a protein. In Silico Biol. 2002;2:291-303.

12. Wang MJ, Yang J, Xu ZJ, Chou KC. SLLE for predicting membrane protein types. J Theor Biol. 2005;232:7-15.

13. Wang SQ, Yang J, Chou KC. Using stacked generalization to predict membrane protein types based on pseudo amino acid composition. J Theor Biol. 2006;242:941-946.

14. Wang M, Yang J, Liu GP, Xu ZJ, Chou KC. Weighted-support vector machines for predicting membrane protein types based on pseudo amino acid composition. Protein Eng Des Sel. 2004;17:509-516.
15. Zhang SW, Pan Q, Zhang HC, Shao ZC, Shi JY. Prediction protein homo-oligomer types by pseudo amino acid composition: Approached with an improved feature extraction and naive Bayes feature fusion. Amino Acids. 2006;30:461-468.

16. Gao Y, Shao SH, Xiao H, et al. Using pseudo amino acid composition to predict protein subcellular location: approached with Lyapunov index, Bessel function, and Chebyshev filter. Amino Acids. 2005;28:373-376.

17. Guo YZ, Li M, Lu M, et al. Classifying G protein-coupled receptors and nuclear receptors based on protein power spectrum from fast Fourier transform. Amino Acids. 2006;30:397-402.

18. Liu H, Wang M, Chou KC. Low-frequency Fourier spectrum for predicting membrane protein types. Biochem Biophys Res Comm. 2005;336: 737-739.

19. Chou KC. Prediction of protein subcellular locations by incorporating quasi-sequence-order effect. Biochem Biophys Res Comm. 2000;278: $477-483$.

20. Chou CK. A novel approach to predicting protein structural classes in a (20-1)-D amino acid composition space. Proteins. 1995;21:319-344.

21. Chou KC, Zhang CT. Predicting protein folding types by distance functions that make allowances for amino acid interactions. J Biol Chem. 1994;269:22014-22020.

22. Chou KC, Zhang CT. Review: Prediction of protein structural classes. Crit Rev Biochem Mol Biol. 1995;30:275-349.

23. Chou KC, Elrod DW. Protein subcellular location prediction. Protein Eng. 1999;12:107-118.

24. Chou KC. Review: Prediction of protein structural classes and subcellular locations. Curr Protein Pept Sci. 2001;1:171-208.

25. Chou KC, Elrod DW. Prediction of membrane protein types and subcellular. Locations. Proteins. 1999;34:137-153.

26. Chou KC, Elrod DW. Prediction of enzyme family classes. J Proteome Res. 2003;2:183-190.

27. Chou KC, Cai YD. Predicting enzyme family class in a hybridization space. Protein Sci. 2004;13:2857-2863.

28. Chou KC, Elrod DW. Bioinformatical analysis of G-protein-coupled receptors. J Proteome Res. 2002;1:429-433.

29. Chou KC. Prediction of G-protein-coupled receptor classes. J Proteome Res. 2005;4:1413-1418.

30. Chou KC, Cai YD. Prediction of protease types in a hybridization space. Biochem Biophys Res Comm. 2006;339:1015-1020.

31. Chou KC, CaiYD. Predicting protein-protein interactions from sequences in a hybridization space. J Proteome Res. 2006;5:316-322.

32. Chou KC, Cai YD, Zhong WZ. Predicting networking couples for metabolic pathways of arabidopsis. EXCLI Journal. 2006;5:55-65.

33. Chou KC, Cai YD. Predicting protein quaternary structure by pseudo amino acid composition. Proteins. 2003;53:282-9.

34. Kuri L. The digital language of amino acids. Amino Acids. 2007; 653-661.

35. Kuri L. The atomic genetic code. J Comput Biol. 2009;2:101-16.

36. Kuri L. Mesure complexe des caracteristiques dynamiques de series temporelles. Journal de la Societe de statistique de Paris. tome 127, No 2.1986.

\section{Publish your work in this journal}

Advances and Applications in Bioinformatics and Chemistry is an international, peer-reviewed open-access journal that publishes articles in the following fields: Computational biomodelling; Bioinformatics; Computational genomics; Molecular modelling; Protein structure modelling and structural genomics; Systems Biology; Computational

\section{Dovepress}

Biochemistry; Computational Biophysics; Chemoinformatics and Drug Design; In silico ADME/Tox prediction. The manuscript management system is completely online and includes a very quick and fair peerreview system, which is all easy to use. Visit http://www.dovepress.com/ testimonials.php to read real quotes from published authors. 